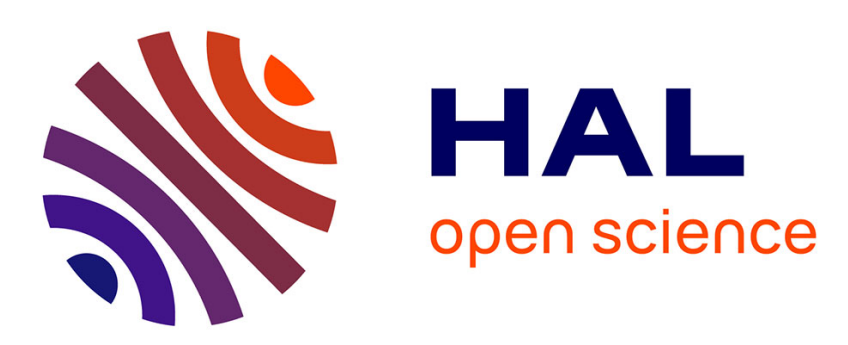

\title{
Design, modeling and control of a modular contactless wafer handling system.
}

Bassem Dahroug, Guillaume Laurent, Valerian Guelpa, Nadine Piat

\section{To cite this version:}

Bassem Dahroug, Guillaume Laurent, Valerian Guelpa, Nadine Piat. Design, modeling and control of a modular contactless wafer handling system.. International Conference on Robotics and Automation, May 2015, Seattle, United States. hal-02868219

\section{HAL Id: hal-02868219 https://hal.science/hal-02868219}

Submitted on 15 Jun 2020

HAL is a multi-disciplinary open access archive for the deposit and dissemination of scientific research documents, whether they are published or not. The documents may come from teaching and research institutions in France or abroad, or from public or private research centers.
L'archive ouverte pluridisciplinaire HAL, est destinée au dépôt et à la diffusion de documents scientifiques de niveau recherche, publiés ou non, émanant des établissements d'enseignement et de recherche français ou étrangers, des laboratoires publics ou privés. 


\title{
Design, modeling and control of a modular contactless wafer handling system
}

\author{
Bassem Dahroug, Guillaume J. Laurent, Valérian Guelpa and Nadine Le Fort-Piat
}

\begin{abstract}
In the photovoltaic solar cell industry as in the semiconductor industry, efforts to reduce the thickness of silicon wafers are in progress. Wafer damage and breakage during handling can lead to unacceptable yields and alternative solutions have to be proposed. This paper presents a modular contact-free wafer handling system that responds to the industrial requirements in terms of throughput and flexibility. The system is based on simple unidirectional modules that can be assembled together to form the desired trajectory. A complete and accurate physical model of the modular system describing the motion of the wafer transported by directed airjets is proposed. A decentralized control at the block level is realized to damp the object motion. The experimental results show a great reduction of the response time compared to free motion and a standard deviation of the servo error below the millimeter. In addition, simulations show that a $150 \mathrm{~mm}$ wafer could reach a speed up to $2.9 \mathrm{~m} / \mathrm{s}$ on large conveyors.
\end{abstract}

\section{INTRODUCTION}

The photovoltaic (PV) solar cell industry and the semiconductor industry are making efforts to reduce the thickness of silicon wafers while increasing their size to achieve cost reduction and cycle time improvement in manufacturing.

However large and thin wafers are delicate and notoriously difficult to handle without inducing damage in the form of chipping and cracking [1]. Recent industrial studies have shown that the use of thinner wafers can lead to unacceptable yields arising from wafer and cell breakage due to transport during production [2]. Indeed, as wafer thickness is decreasing, wafers are less stable and more vulnerable to stresses, and grippers can be prone to breaking and scratching especially during handling between machines [3].

Low-cost thin wafer handling is one of the difficult challenges that has been identified in the 2013 International Technology Roadmap For Semiconductors Roadmap [4].

Conventional wafer-transfer systems use comb pairs to lift wafers out of carriers, comb assemblies to retain the wafers, Bernoulli grippers to handle and to separate wafers, and wheel conveyors. As wafers become thinner and cycle time shorter, these conventional methods become problematic. The low thickness of the wafers and their sharp edges make it difficult to handle, which ultimately can cause wafer breakage [1].

Another class of handling method employs air jets to position the wafers and transfer them to processing stations without ever touching them.

This work was supported in part by the Smart Blocks project (ANR-2512011-BS03-005), by the Labex ACTION project (ANR-11-LABX-01-01) and by the Franche-Comté région. B. Dahroug, G. J. Laurent, V. Guelpa and N. Le Fort-Piat are with the FEMTO-ST Institute, UFC-ENSMM-UTBMCNRS, Université de Franche-Comté, Besançon, France, Corresponding author: guillaume. laurent@ens $2 \mathrm{~m}$. fr
For instance, air bearings are widely used to transport large, thin and heavy products like glass and LCD panels [5]. Instead of pressurized air fed through orifice or porous media, ultrasound bearings can also be used to lift a substrate over a vibrating plate [6], [7], [8], [9]. But air bearing and vibrating tables realize only the levitation of product, the motion is still achieved by another principle (gravity, wheels, belt conveyors).

To move wafers, a set of inclined holes can create an air flow in addition to the air cushion under the object. Combination of several nozzles with different orientation can produce different functions such as transfer track, position control track, orientation control track, etc.

The first attempts to use this principle to transport and position wafers were in Texas Instruments by Hagler et al. [10] and in IBM by Hassan and Paivanas [11], [12]. Toda et al. [13], [14] improved the concept and made a transfer system for $300 \mathrm{~mm}$ wafer. The wafer track consists of a perforated plate with $0.5 \mathrm{~mm}$-diameter holes. Transport times of less than $15 \mathrm{sec}$, from wafer moving at $0.12 \mathrm{~m} / \mathrm{s}$ to wafer stopping, were achieved in the $0.8 \mathrm{~m}$-long wafer transport track.

Similar systems have been investigated by Moon and Hwang [15] and by Kim and Shin [16], [17]. Kim and Shin proposed some methods to compute the precise position on the wafer with cheap photo proximity sensors but no experimental performances are reported.

In [18], [19], Wesselingh et al. introduced a new concept of wafer positionner based on an array of cells able to generate an air film for both suspension and motion. Their device is dedicated to wafer micro-positioning. Experiments were realized with low cost reflectance based optical sensors to detect the edge of the wafer. A servo error of $20 \mu \mathrm{m}$ (P-P) and a positioning repeatability of $3 \mu \mathrm{m}$ (STD) were reported [20].

Luntz and Moon [21], [22] proposed another principle based on potential air flow to move an object on an airhockey table. Their method allows to move an object to a unique final pose using airflow fields without sensors. Laurent et al. [23], [24] used the proposed traction principle to move a product thanks to an array of vertical air jets which induce desired potential air flow over the surface. This device is able to move centimeter-sized objects up to $0.2 \mathrm{~m} / \mathrm{s}$ with millimetric closed-loop positioning accuracy.

All these devices are designed to achieve a dedicated task whereas industries require more flexibility and efficiency. In addition, the proposed solutions are too slow to meet industrial high throughput. 
In this paper, we introduce a novel concept of contactfree conveyor of wafers for the PV and the semiconductor industries. At the opposite of devices dedicated to one task and motion, we propose a modular approach based on simple unidirectional modules that can be assembled together to form the desired function. This approach allows cost reduction and the making of various and large conveyors.

This paper presents the concept and the design of a contact-free modular conveyor along with a complete physical model of the dynamics of the wafer moving on the conveyor. Then a decentralized control is proposed to damp the free oscillation of the wafer. After the experimental validation, we finally explore the potentiality of decentralized control applied to large conveyors.

\section{Modular Design}

\section{A. Modular concept}

Conveyors are usually designed as monolithic entities solving one problem at one time. A standard conveying belt is able to convey jumbled objects but it can neither position nor sort them. A conveyor made of modules, blocks, will allow the integration of different kinds of functionalities inside one single design. The idea is that complex conveyors can be made by the assembly of several simple blocks.

On the one hand, having identical and modular blocks decreases the complexity of each block which makes them easier and cheaper to produce. On the other hand, designing too small modules could dramatically increase the complexity and the cost of a conveyor. Thus, the size of the blocks must be adapted to the dimensions of transported objects. The goal is to design the largest modules without loosing flexibility.

To reduce the cost of production, the blocks must also have a very simple design. In this paper, we focus of the conveyance of circular wafers using air flow. Among the mentioned possibilities to move an object on a air cushion, an array of titled air jet is the simplest solution. That is why we propose to use square blocks which upper surfaces are drilled of tilted holes with the same orientation. Indeed, a single block can push the wafer in a determined direction. The assembly of unidirectional blocks can generate various motion of the wafer.

A block side equal to the half of the wafer diameter is the best compromise between flexibility and cost. This size allows to fullfill all the required functionalities of complex conveyors such as moving, guiding, positioning and rotating as shown in the Figure 1.

\section{B. Block Design}

In this paper, we used $150 \mathrm{~mm}$ circular wafers to demonstrate the potentiality of the approach but larger wafers could be handle as well.

To handle $150 \mathrm{~mm}$ circular wafers, the blocks must have a dimension of $75 \times 75 \mathrm{~mm}$. The block was fabricated by a 3D printer, as shown in Figure 2a. The upper surface of the block contains 128 holes with a tilt angle of $45^{\circ}$. The diameter of each jet is preferred to be $0.5 \mathrm{~mm}$ because of the

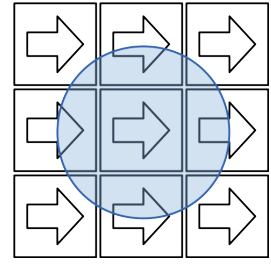

(a) Simple motion

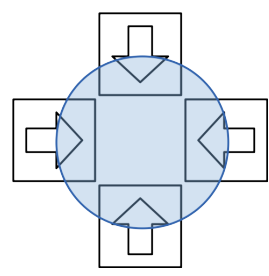

(c) Positioning

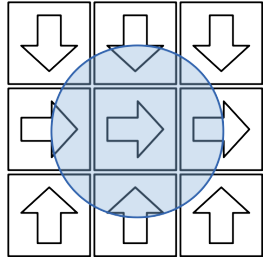

(b) Guided motion

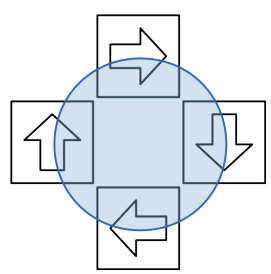

(d) Rotation
Fig. 1. Illustration of the modular concept.

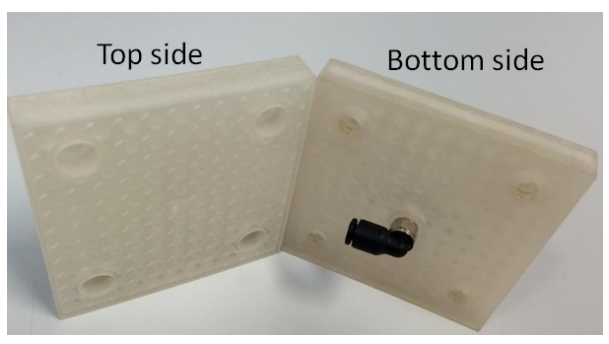

(a) 3D printed modules

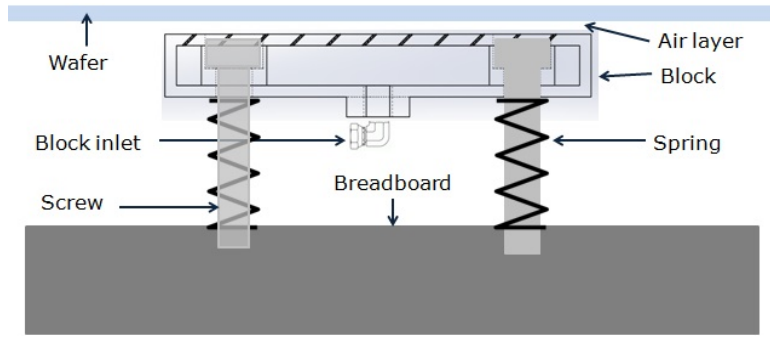

(b) Cross view of a module

Fig. 2. Design of the blocks.

resolution of the 3D printer. Each block is driven by a $3 / 2$ pneumatic proportional valve. The blocks are attached on a breadboard using four screws and four springs in order that the block surface can be adjusted to be perfectly horizontal, as illustrated in Figure 2b.

\section{Physical Modeling and Parameter IDENTIFICATION}

In this section we proposed a physical modeling of the force exerted by a block on a moving wafer. After the identification of the propulsion coefficient, the model is validated with an experimental trial.

\section{A. Physical Modeling}

The wafer is propelled by the action of tilted air-jets (cf. Figure 3). A synthetic model of air-jets has been proposed by Toda et al. [13]. The tangential force $F_{P}$ exerted by a 


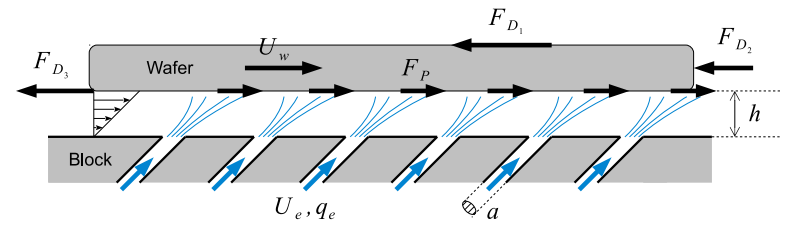

Fig. 3. Principle and modeling of tilted air-jet surfaces.

sole covered jet onto the wafer can be calculated according to a drag force equation:

$F_{P}=\frac{1}{2} \rho C_{P} a \sin \theta\left(U_{e}-U_{w}\right)^{2}=\frac{1}{2} \rho C_{P} a \sin \theta\left(\frac{q_{e}}{a}-U_{w}\right)^{2}$

where $C_{p}$ is a dimensionless propulsion coefficient, $\rho$ the fluid density, $\theta$ the inclination angle of the holes from the vertical, $a=\pi r_{i}^{2}$ the section area of a hole, $U_{e}$ the exit speed of gas in nozzle, $q_{e}=a U_{e}$ the supplied gas flow rate per each hole, and $U_{w}$ the speed of the wafer over the hole and in the direction of the jet.

The total propulsive force is directly proportional to the number $k$ of air jets which are covered by the wafer.

When moving on the air-bearing, the substrate is slow down by the air resistance. The resistance force is the sum of the frictional resistance acting on the upper surface, the drag force acting on the edge, and the frictional resistance acting on the underside (cf. Figure 3).

In the case of wafer handling on a thin air-film, the upper frictional resistance and the drag force are negligible with regards to the underside frictional resistance. This force can be described by a Couette's flow and calculated by:

$$
F_{D}=\frac{\mu \pi r_{d}^{2}}{h} U_{w}
$$

where $\mu$ is the dynamic viscosity of air, $r_{d}$ the radius of the disk, and $h$ the levitation height.

Finally, the equation of the motion of the wafer is:

$$
m \frac{d U_{w}}{d t}=k * F_{P}-F_{D}
$$

where $m$ is the wafer's mass.

The remaining problem is to calculate the levitation height $h$. The pressure distribution below the wafer is very complex in general. Even for simple case such as a disk centered on a single hole, an exact solution of the Navier-Stokes equation appears to be difficult. McDonald proposed an approximate solution for a disk centered on a single hole assuming that the velocity is purely radial [25]. The approximated aerostatic lifting force is given by:

$$
F_{L}=\frac{3 \mu Q_{e} \pi r_{d}^{2}}{\pi h^{3}}=m g
$$

This equation stands for a disk centered on a single vertical hole on a thin air film but experiments showed that it is also a reasonable approximation for different shapes and tables with multiple tilted holes. In this case, $Q_{e}$ is simply the sum of the flow of the air jets that impinge on the underside of the object.

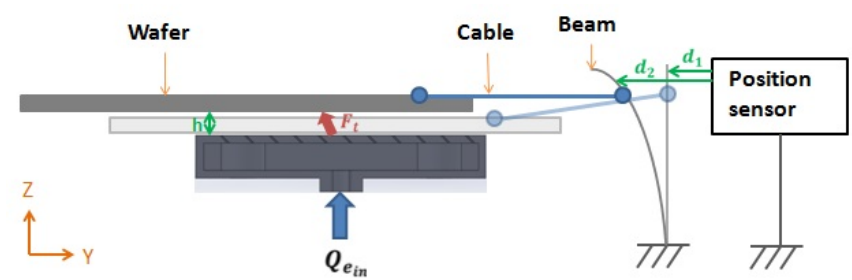

(a) Cross view

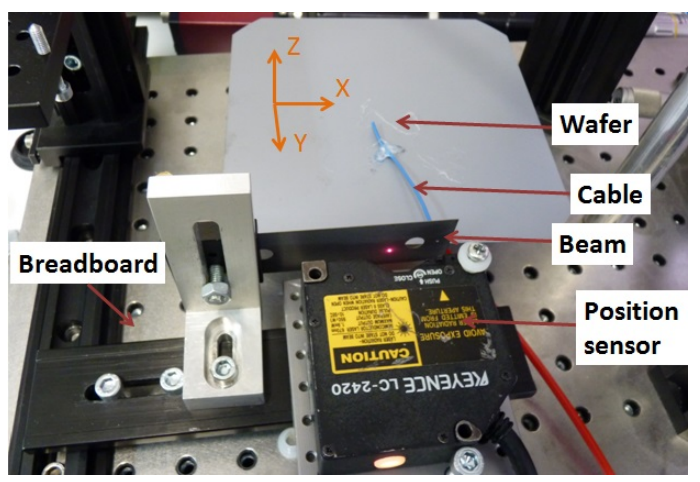

(b) Top view

Fig. 4. Experimental setup for propulsion coefficient identification.

\section{B. Propulsion coefficient identification}

To simulate the motion of the wafer, we need to identify propulsion force according to the air volume flow rate.

a) Experimental setup: A beam of elastic material is fixed alongside a displacement sensor. This sensor measures the displacement $\delta$ of the beam when a force pushes it. Then, the wafer and the beam are tied with a cable in order to allow the vertical motion of wafer, see Figure 4. Finally, the wafer is placed over four blocks to begin the experience.

b) Calibration: Before starting the experiment, the distance $d_{1}$ between the sensor and the beam should be measured. The stiffness coefficient of the beam is also needed to be determined. We used calibrated weights to progressively deform the beam. The beam displacement $\delta=d_{2}-d_{1}$ is measured in function of the weights added. A linear relation between the displacement and the force is obtained since $F=K_{b} \delta . K_{b}$ is the beam stiffness and is equal to 348.8.

c) Determination of the propulsion coefficient $C_{p}$ : The horizontal motion of the wafer is constrained by the beam. Therefore, the summation of horizontal forces is equal to zero when the wafer velocity is null along the $\mathrm{X}$ axis. So, the propulsion coefficient can be calculated rewriting equation 1 :

$$
C_{p}=\frac{2 a K_{b} \delta}{\rho k q_{e}^{2} \sin \theta}
$$

A comparison between the experimental results of the propulsion force and the analytic model is presented in Figure 5 which shows how the two results are homogeneous and in agreement. According to these experimental results, the value of $C_{p}$ is calculated when $k=128 \times 4$ holes, $\rho=1.2041 \mathrm{~kg} / \mathrm{m}^{3}, r_{i}=0.00025 \mathrm{~m}$ and $\theta=45^{\circ}$. The average value of $C_{p}$ is 12.1 and it is independent of levitation height. 


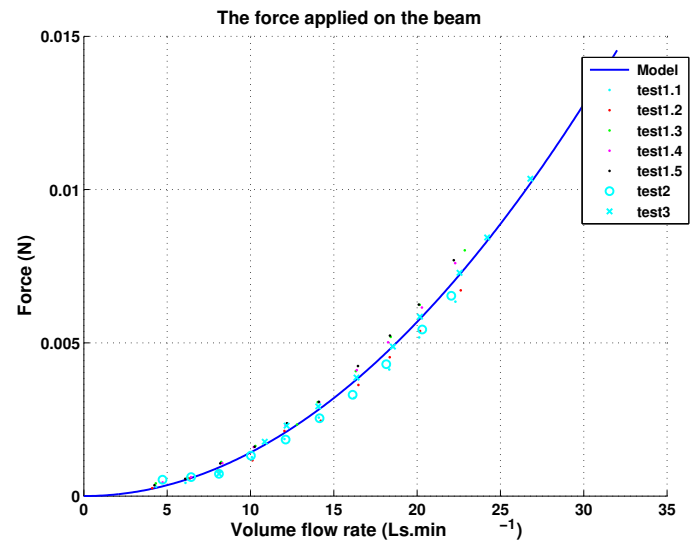

Fig. 5. Results of the force applied on the beam, in function of the total volume flow rate (the first test series ', was done with a wafer of mass $11.69 * 10^{-3} \mathrm{~kg}$, the second test series ' $*$, with a mass of $13.77 * 10^{-3} \mathrm{~kg}$ and the last 'o' with a mass of $20.38 * 10^{-3} \mathrm{~kg}$ ).

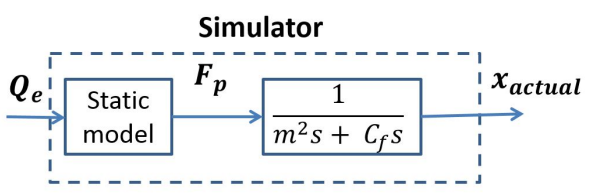

Fig. 6. Simulator block diagram.

\section{Model Validation}

A simulator has been implemented using $\mathbf{C + +}$ in a $\mathrm{C}$ MEX Simulink function. It is based on the analytical model of Section III-A, where its input is the volume flow rate for each block and its output is the wafer position in XY plane. The simulator block diagram is presented in Figure 6. The transfer functions of the dynamic model derived from Equation 3 is:

$$
G(s)=\frac{X(s)}{F_{p}(s)}=\frac{1}{m s^{2}+C_{f} s}
$$

where $C_{f}$ is a constant that groups the coefficients of friction.

To validate the simulator in comparison with a practical case, a modular conveyor was built using nine passive blocks, where the air flow rate is constant for each block. The blocks arrangement is shown in Figure 7. This configuration allows to move the wafer towards the center of the manipulator. Therefore, the wafer trajectory over the blocks is traced in two cases, where the first case is done with the simulator (one-step ahead prediction) and the second one is done practically. The Figure 7 shows the comparison between these two cases.

The results of the simulation are very close the to experimental points. The standard deviation of the prediction error is $0.64 \mathrm{~mm}$ in the $\mathrm{X}$ direction and $0.95 \mathrm{~mm}$ in the $\mathrm{Y}$ direction.

It is noteworthy that the wafer motion is not well stabilized at a fixed point. In fact, the wafer moves continuously in a large region because of the very low damping. As a

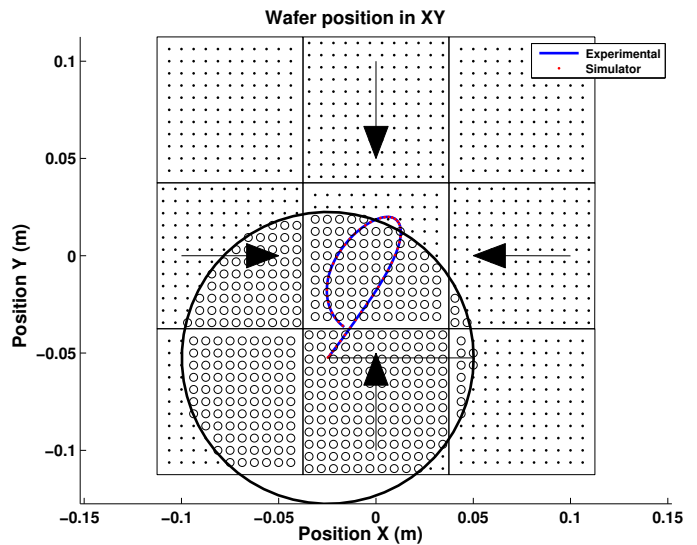

Fig. 7. Block setup and comparison between the wafer trajectory in the simulator and in the practical experimental.
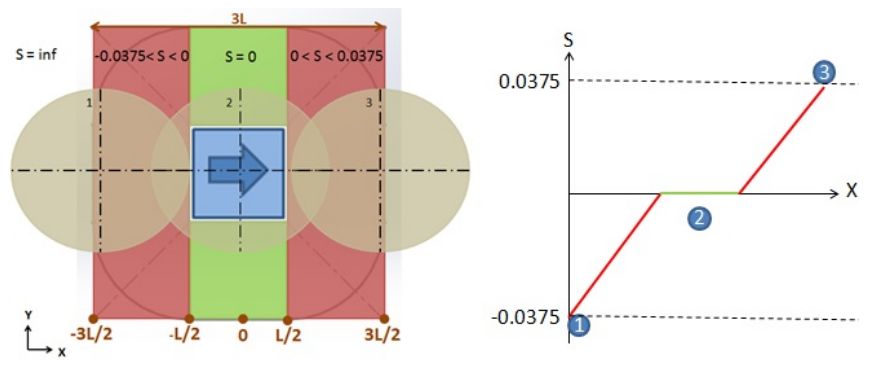

Fig. 8. Diagram of the region formed by the position sensor and its output response.

consequence, it is required to control the motion of wafer in closed loop in order to improve the system response.

\section{Decentralized CONTROL}

The conveying surface is formed from blocks where each block have its own microcontroller, actuator (pneumatic valve) and wafer detectors. Each block is supposed to be capable on it is own to locate and to move an object above its surface.

\section{A. Position control}

Each block will deduce the wafer position with respect to its position using its wafer detectors. The position of the wafer will be represented by the control variable $S$ which equals to the surface of the block being covered by the wafer. This information will be interpreted by the microcontroller to form a region around the block where it can detect the wafer. The Figure 8 shows the different regions and the expected values of $S$.

More precisely, the sensor coverage value $S$ can be represented by the following equation, where $x_{w}$ is the wafer center position in $\mathrm{X}$ axis and $L$ is the side block width:

$$
S=\left\{\begin{array}{cc}
\infty & \text { if } x_{w}<\frac{-3 L}{2} \text { or } x_{w}>\frac{3 L}{2} \\
0 & \text { if } \frac{-L}{2} \leq x_{w} \leq \frac{L}{2} \\
x_{w}-\frac{L}{2} & \text { if } \frac{-3 L}{2} \leq x_{w}<\frac{-L}{2} \\
x_{w}+\frac{L}{2} & \text { if } \frac{3 L}{2} \leq x_{w}<\frac{L}{2}
\end{array}\right.
$$




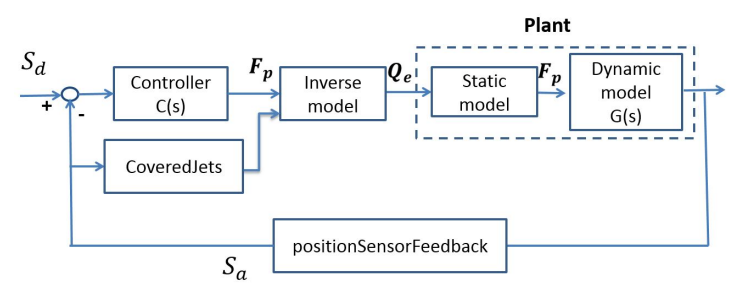

Fig. 9. Block diagram of decentralized control.

\section{B. Inverse modeling control}

The system block diagram is represented in Figure 9, where the plant analytical model can be divided into static and dynamic models. The static model is given by equation (1) and links the air volume flow rate to the applied propulsion force. From this equation, the propulsion force is directly proportional with the square of the volume flow rate. This force is also proportional with the number of jet covered by the wafer. The dynamic model relies the propulsion force to the wafer position. This model is a common mechanical system of second order.

The inverse model is used to compensate the non linearity of the static part of the plant. The inverse model gives the required air volume flow rate to obtain the force calculated by the controller. Since the inverse model compensate the non linearity of the system, the controller just need to compensate a second-order linear system.

\section{Controller synthesis}

The foreseen microcontroller is not very powerful in term of memory size and compilation speed. For this reason, it was decided to implement a PD control law.

The control loop of Figure 9 can be simplified by eliminating the static non-linear part of the plant with the inverse model. After the simplification, the loop is composed of the PD controller, the dynamic part of the plant and the unity feedback. The transfer functions of the controller is presented in the following equation, where $k_{p}$ is the constant of the proportional controller and $k_{d}$ is the constant of the derivative controller:

$$
C(s)=\frac{F_{p}(s)}{E(s)}=k_{p}+k_{d} s
$$

The global transfer function of the system can be presented by:

$$
H(s)=\frac{C(s) G(s)}{1+C(s) G(s)}=\frac{1}{m} * \frac{k_{p}+k_{d} s}{s^{2}+\frac{C_{f}+k_{d}}{m} s+\frac{k_{p}}{m}}
$$

From this last equation, values of $k_{p}$ and $k_{d}$ can be calculated by comparing the denominator of $H(s)$ with the denominator of a mechanical system of second order. By using pole placement method, $\xi$ and $\omega_{n}$ are modified in order to obtain a highly damped system $(\xi=1.5)$ and fast response $\left(\omega_{n}=2\right)$. These values also guaranty the stability even when four blocks act on the same wafer.

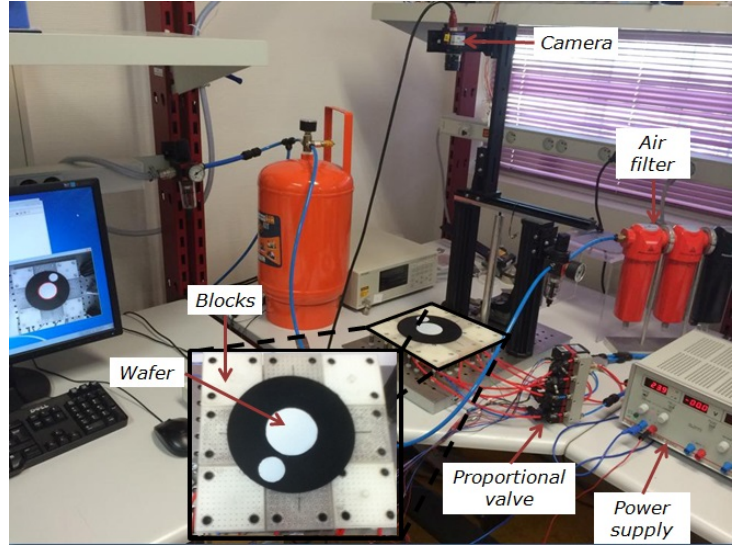

Fig. 10. Setup of the closed-loop experiments.

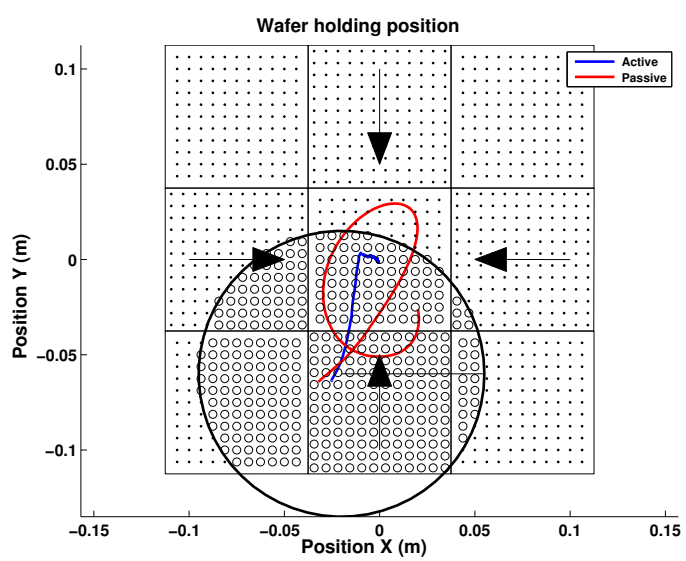

Fig. 11. Comparison between active blocks and passive blocks in $X Y$ plane.

\section{EXPERIMENTAL VALIDATION}

Each block is able to control the position of an object above its surface. In this section, this decentralized control is validated by doing an experiment with four active blocks.

The setup consists in five passive blocks to maintain the levitation height and four active blocks to move the disc in the $X Y$ plane. Each active block has its own controller to take its decision but a camera is used to emulate the block position sensors. The experimental setup is presented in Figure 10.

The objective of this test is to hold the position of a glass wafer at the center of the handling surface. The wafer has a mass of $13.23 \mathrm{~g}$ and a diameter of $150 \mathrm{~mm}$. The first test is done when all blocks are passive (no closed-loop control). The input flow rate of air is set to $7.5 \mathrm{Ls} / \mathrm{min}$ for each block. The second test is done from the same initial point but when the four blocks are active (decentralized closed-loop control). Figure 11 shows the position of the wafer at the beginning of the experiment and the trajectory of the wafer in the passive case (red line) and in the active case (blue line).

These results show the interest of active control which is faster to achieve the stability at a point. The active system that reaches the center in $1 \mathrm{~s}$ in $\mathrm{X}$ direction and $1.1 \mathrm{~s}$ in $\mathrm{Y}$ direction. In addition, the standard deviation of the position error in $\mathrm{X}$ and $\mathrm{Y}$ are $0.19 \mathrm{~mm}$ and $0.34 \mathrm{~mm}$ respectively. 


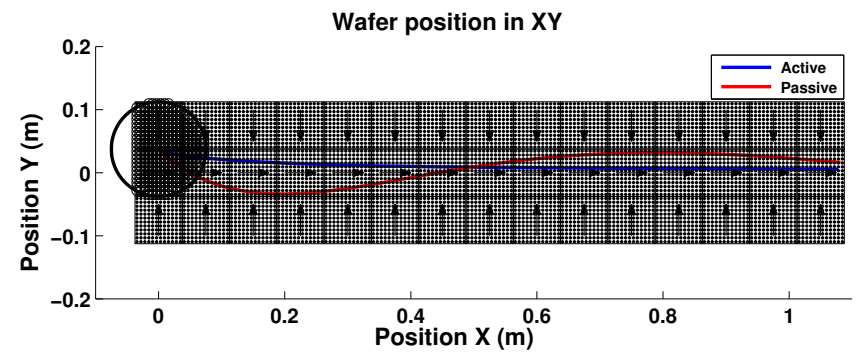

Fig. 12. Blocks arrangement and comparison between the response of active conveyor and passive conveyor.

\section{Design of LARGe aCtive CONVEYors}

Thanks to the experimental validation, we can apply the same concept to larger surfaces using simulation. To illustrate the capability of the modular system to transport wafer over a long distance, we designed a $1 \mathrm{~m}$-long track made of 45 blocks.

The system response is compared in two cases. The first one when all blocks are passive (passive conveyor) and the second when blocks on the edge are active, where the air volume flow rate per block is $7.5 \mathrm{Ls} / \mathrm{min}$. On the one hand, the system response in the passive case is presented is in Figure 12, with the red line, where the wafer oscillates in the $\mathrm{Y}$ direction. On the other hand, in the active case, the wafer's oscillation in the $\mathrm{Y}$ direction is removed and the wafer reaches stability faster, as shown the blue line in Figure 12. Also, the wafer starts from rest and it arrives at the conveyor end with a velocity of $0.62 \mathrm{~m} / \mathrm{s}$.

On even larger conveyor, the speed of the wafer could theoretically reaches $2.9 \mathrm{~m} / \mathrm{s}$ with a the maximal acceleration of $0.48 \mathrm{~m} / \mathrm{s}^{2}$.

\section{CONCLUSION}

In this paper, we presented the design and the implementation of an active modular conveying surface for transporting wafers on an air cushion to avoid any contact.

We proposed a complete physical model of the modular system describing the motion of the wafer transported by directed air-jets. Experimental comparisons show that the model predicts accurately the motion of the wafer according to the arrangement of the blocks and the volume air flow. Based on this model, we synthesized a decentralized control at the block level to damp the object motion. The experimental results show a great reduction of the response time and a standard deviation of the position error below the millimeter.

Finally, we showed the interest of the modular approach for making large conveyor. For instance, a $150 \mathrm{~mm}$ wafer could reach a speed up to $0.64 \mathrm{~m} / \mathrm{s}$ on a $1 \mathrm{~m}$-long acceleration track. Maximal operational speed is calculated to be $2.9 \mathrm{~m} / \mathrm{s}$.

Future work concerns the trajectory planning. Indeed, when the desired trajectory becomes long and curved, when handling multiple wafers at the same time, and also to increase the system efficiency, an optimizing algorithm is required to find the best arrangement of the blocks.

\section{REFERENCES}

[1] L. Teschler. Next big challenge for PV makers: Wafer handling. Machine Design, 2008.

[2] X. F. Brun and S. N. Melkote. Analysis of stresses and breakage of crystalline silicon wafers during handling and transport. Solar Energy Materials \& Solar Cells, 93:1238-1247, 2009.

[3] J. N. Burghartz, editor. Ultra-thin Chip Technology and Applications. Springer, 2011.

[4] Assembly and Packaging Difficult Challenges. International Technology Roadmap For Semiconductors, 2013.

[5] M. Hoetzle, T. Dunifon, and L. Rozevink. Glass transportation system. U.S. Patent 6,505,483, 2003.

[6] G. Reinhart, M. Heinz, J. Stock, J. Zimmermann, M. Schilp, A. Zitzmann, and J. Hellwig. Non-contact handling and transportation for substrates and microassembly using ultrasound-air-film-technology. In Proc. of the IEEE/SEMI Advanced Semiconductor Manufacturing Conf., pages 1-6, 2011.

[7] J. Höppner and J. Zimmermann. Device for contactlessly gripping and positioning components, November 2003. US Patent 6647791.

[8] M. Schilp, J. Zimmermann, and A. Zitzmann. Device for non-contact transporting and holding of objects or material, December 2011. US Patent 2011/0311320.

[9] J. Zimmermann, D. Jacob, and A. Zitzmann. Device for conveying and positioning of structural elements in non-contact way, August 2007. US Patent 7260449.

[10] R. G. Hagler. Transporting and positioning system, 1973. US Patent 3717381 .

[11] J. K. Hassan and J. A. Paivanas. Pneumatic control of the motion of objects suspended on an air film, 1979. US Patent 4165132.

[12] J. A. Paivanas and J. K. Hassan. Air film system for handling semiconductor wafers. IBM Journal of Research and Development, 23(4):361-375, 1979.

[13] M. Toda, T. Ohmi, T. Nitta, Y. Saito, Y. Kanno, M. Umeda, M. Yagai, and $\mathrm{H}$. Kidokoro. $\mathrm{N}_{2}$ tunnel wafer transport system. Journal of the Institute of Environmental Sciences, 40(1):23-28, 1997.

[14] M. Toda, M. Umeda, Y. Kanno, and T. Ohmi. Floating apparatus of substrate, May 2000. EP Patent 1005076.

[15] I.-H. Moon and Y.-K. Hwang. Evaluation of a wafer transportation speed for propulsion nozzle array on air levitation system. Journal of Mechanical Science and Technology, 20(9):1492-1501, 2006.

[16] Y.-J. Kim and D. H. Shin. Wafer position sensing and motion control in the clean tube system. In Proc. of the IEEE Int. Conf. on Industrial Technology, pages 1315-1319, 2006.

[17] D. H. Shin, H. G. Lee, and H. S. Kim. Wafer positioning control of clean tube system. In Proc. of the ACSE Conf., 2005.

[18] J. Wesselingh, R.A.J. van Ostayen, J.W. Spronck, R.H.Munnig Schmidt, and J. van Eijk. Actuator for contactless transport and positioning of large flat substrates. In Proc. of the EUSPEN Int. Conf., 2008.

[19] J. van Rij, J. Wesselingh, R. A. J. van Ostayen, J.W. Spronck, R.H. Munnig Schmidt, and J. van Eijk. Planar wafer transport and positioning on an air film using a viscous traction principle. Tribology International, 42:1542-1549, 2009.

[20] J. Wesselingh, J.W. Spronck, R.A.J. van Ostayen, and J. van Eijk. Contactless 6 dof planar positioning system utilizing an active air film. In Proc. of the EUSPEN Int. Conf., 2010.

[21] J. Luntz and H. Moon. Distributed manipulation with passive air flow. In Proc. of the IEEE/RSJ Int. Conf. on Intelligent Robots and Systems, pages 195-201, 2001.

[22] H. Moon and J. Luntz. Distributed manipulation of flat objects with two airflow sinks. IEEE Transactions on robotics, 22(6):1189-1201, 2006.

[23] G. J. Laurent, A. Delettre, and N. Le Fort-Piat. A new aerodynamic traction principe for handling products on an air cushion. IEEE Transactions on robotics, 27(2):379-384, 2011.

[24] A. Delettre, G. J. Laurent, Y. Haddab, and N. Le Fort-Piat. Robust control of a planar manipulator for flexible and contactless handling. Mechatronics, 22(6):852-861, 2012.

[25] K. T. McDonald. Radial viscous flow between two parallel annular plates. arXiv:physics/0006067, 2000. 